\title{
Dentin dysplasia-sclerotic bones syndrome
}

INSERM

\section{Source}

INSERM. (1999). Orphanet: an online rare disease and orphan drug data base. Dentin dysplasia-sclerotic bones syndrome. ORPHA:99792

Dentin dysplasia-sclerotic bones syndrome is a rare, genetic odontologic disease

characterized by the clinical, radiog raphic, and histologic features of dentine dysplasia and osteosclerosis of all long bones, with heavy cortical bone and narrowed or occluded marrow spaces. There have been no further descriptions in the literature since 1977. 